\title{
Comunicación

\section{Evaluación del desempeño de los medios informativos en las elecciones de 2009 en Monterrey}

Voters' assessment about media electoral coverage of the 2009 election

for governor in Monterrey

JosÉ-CARLOS LOZANO, JESÚS CANTÚ, FRANCISCO JAVIER MARTÍNEZ, CINTIA SMITH ${ }^{1}$

Poco se sabe sobre los usos y lecturas de las noticias electorales por parte de votantes mexicanos. Los resultados de esta encuesta telefónica por muestreo en Monterrey, Nuevo León, discutidos desde la perspectiva de los estudios culturales, indican que los votantes de esa ciudad consideraron objetiva, balanceada y útil la cobertura de sus medios informativos sobre la campaña electoral a la gubernatura del Estado de Nuevo León de 2009.

PALABRAS ClAVE: Audiencias, cobertura electoral, estudios culturales, comunicación política, credibilidad y uso de información política.
This work presents the results of a telephone survey in the metropolitan area of Monterrey (Mexico), in which respondents assessed newspapers and television news programs' coverage of the 2009 electoral campaign to the governorship of the state of Nuevo León. Surveyed participants found media coverage was objective, balanced and useful to inform their knowledge of the political alternatives.

KEY WORDS: Electoral news, audiences, cultural studies, credibility and uses of political news.

1 Instituto Tecnológico y de Estudios Superiores de Monterrey, México.

Correos electrónicos: jclozano@itesm.mx; jce@itesm.mx;

francisco@itesm.mx; csmith@itesm.mx

Av. Eugenio Garza Sada no. 2501 Sur, Col. Tecnológico Monterrey, C.P. 64849; Monterrey, N.L., México. 
Los estudios sobre cómo leen y dan sentido a las noticias electorales los miembros de las audiencias tienen una tradición amplia y consistente en el campo liberal-pluralista de la comunicación política (Abbe, Goodliffe, Herrnson \& Patterson, 2003; Colwell, 1979; David, 2009; De Vreese, 2004; Eveland, Shah \& Kwak, 2003; Graber, 1993; Rhee \& Capella, 1997) aunque no en la investigación basada en la perspectiva de los estudios culturales, la cual se ha centrado principalmente en la recepción de noticias generales (Gauntlett y Hill, 1999; Hacker, Coste, Kamm \& Bybee, 1991; Jensen, 1998; Madianou, 2005; Morley, 1980; Orozco, 1997; Orozco de Medina \& Jackson, 2000; Sigman \& Fry, 1985). Al interpretar los hallazgos de una encuesta telefónica ${ }^{2}$ a votantes en las elecciones para gobernador de Nuevo León de 2009, este trabajo ofrece evidencias, desde una aproximación vinculada a esta última perspectiva (los estudios culturales), sobre la percepción y evaluación por parte de lectores y televidentes del desempeño de los diarios y los noticieros televisivos locales al cubrir una campaña electoral de carácter local.

\section{INVESTIGACIÓN PREVIA \\ SOBRE NOTICIAS ELECTORALES}

Diversos politólogos y estudiosos de la comunicación política afirman que en los países occidentales la tasa de participación electoral, la identificación partidista y la participación política tradicional se encuentran decreciendo, mientras que la desconfianza hacia los políticos y las instituciones políticas se incrementan (Hollander, 2007; Putnam, 2000; Strömbäck \& Kaid, 2008; Van Zoonen, 2005). Para Dahlgren y Gurevitch (2005), la tendencia hacia el desinterés y la apatía política

2 El presente trabajo se basa en una encuesta telefónica probabilística realizada por la Cátedra de Investigación en Medios de Comunicación y la Cátedra de Investigación en Instituciones y Prácticas de las Democracias Contemporáneas del Tecnológico de Monterrey, campus Monterrey, con la participación como encuestadores de alumnos de posgrado de las maestrías en Comunicación y en Análisis Político y Medios de Información de la misma universidad. Los investigadores principales del proyecto fueron los cuatro autores de este artículo. 
se mantendrá e incluso se intensificará. De acuerdo a ellos, la desconfianza, ambivalencia e indiferencia, dimensiones de la apatía en el nivel actitudinal, siguen creciendo. Sin embargo, ambos sugieren que esos cambios representarían no un desinterés en la política en sí misma, sino un reenfocamiento de la participación política, o la emergencia de nuevas definiciones de lo que constituye lo político caracterizadas por "una participación personalizada más que colectiva, y un mayor énfasis en temas específicos e individuales que en plataformas o ideologías generales" (pp. 377-378).

Académicos como Farnsworth y Lichter (2007) y Sartori (1998) argumentan que los medios informativos son los culpables de este desinterés y rechazo de la política debido a su poco interés en los temas (issues) al favorecer el drama, la personalización y el entretenimiento en su cobertura de la política y las elecciones. Como explica Van Zoonen (2005), los críticos puntualizan que al proveer:

Info-entretenimiento en lugar de información seria, debates racionales y opiniones sólidas que requieren los ciudadanos para darle sentido a la política, los medios informativos -en particular la televisión- han creado una ciudadanía desinformada, confundida y manipulada que a pesar de eso se siente completamente confiada en sus juicios y elecciones (p. 11).

Para Patterson (citado en Bennett, Rhine \& Flickinger, 2004) el cambio de noticias "duras" a "suaves" y la constante cobertura de cuestiones negativas es la razón del declive en el interés sobre la política entre las audiencias estadounidenses.

En contraste, otros investigadores han encontrado que los medios informativos son realmente útiles en el aprendizaje electoral y político de los votantes y que la exposición a noticias políticas se relaciona con mayores niveles de conocimiento y mayores posibilidades de votar en procesos electorales (David, 2009; Nadeau, Nevitte, Gidengil \& Blais, 2008; Scammell \& Semetko, 2008; Weaver \& Drew, 2001). Otros, sin embargo, arguyen que las visiones tradicionales de la política deberían reemplazarse con definiciones más inclusivas y adecuadas, tomando en cuenta el estudio de otros contenidos mediáticos que pueden ser relevantes, como las películas, las series y programas de televisión 
(Dahlgren \& Gurevitch, 2005; Hermes, 2005; Tenenboim-Weinblatt, 2009; Van Zoonen, 2004, 2007).

Desde la perspectiva de los estudios culturales, trabajos sobre la recepción de noticias generales (no electorales), han demostrado que los lectores y televidentes son activos y capaces de negociar y cuestionar la información que les llega, así como de identificar los intereses políticos o ideológicos de los medios que la difunden (Hacker et al. 1991; Jensen, 1988, 1998; Madianou, 2005; Sigman \& Fry, 1985). Madianou (2005), por ejemplo, en un estudio cualitativo con 35 griegos, chipriotas griegos y turcos viviendo en Atenas, encontró muchas evidencias de cuestionamientos a los contenidos noticiosos y a la forma en que se presentaban. La autora observó, al examinar la recepción de la cobertura sobre asuntos nacionales e internacionales en los medios informativos, que la gente desafiaba el discurso oficial reproducido por las noticias sobre el país y a las noticias mismas.

De igual forma, Hacker, et al. (1991) encontraron que alumnos universitarios eran capaces de identificar sesgos en las noticias televisivas y de considerarlas manipuladoras. Sin embargo, concluyeron que a pesar de esa conciencia sobre los sesgos y la ausencia de objetividad en las noticias, no alcanzaban a percatarse de dimensiones ideológicas más de fondo (p. 198). Sigman y Fry (1985) llegaron a conclusiones muy similares, determinando que mientras que algunos de los lectores de periódicos encuestados por ellos eran capaces de identificar posiciones y sesgos ideológicos, había otros que consideraban a las noticias como factuales, sustantivas y objetivas. Así mismo, advirtieron que al ser requeridos para explicar en sus propias palabras eventos controversiales específicos, los lectores adoptaban claramente los mismos marcos de referencia e incluso la terminología utilizada por el periódico que habían leído, confirmando que los mensajes noticiosos organizan en cierto grado la comprensión de los eventos por parte de las audiencias.

El presente estudio ofrece algunas evidencias sobre el grado en que los votantes de Monterrey, México, percibieron el desempeño de sus medios informativos locales en la cobertura del proceso electoral para la gubernatura del Estado de Nuevo León llevado a cabo de enero a julio de 2009. Incluye también una exploración sobre la influencia de dicha cobertura en la decisión final de voto de los encuestados. Al basarse en datos 
cuantitativos de una encuesta telefónica, el estudio tiene limitaciones en su capacidad de profundizar en los complejos procesos de negociación y apropiación de las noticias electorales por parte de los votantes. Sin embargo, los hallazgos muestran algunas tendencias y percepciones de los votantes sobre los medios informativos y sobre su cobertura electoral que pueden ofrecer pistas relevantes sobre la confianza o desconfianza que las audiencias tienen de los diarios y noticieros televisivos a los que acuden para saber sobre el desarrollo de las campañas electorales.

\section{ELECCIONES ESTATALES EN NUEVO LEÓN, MÉXICO}

El 5 de julio de 2009 se llevaron a cabo las elecciones para gobernador del Estado de Nuevo León, el cual contaba en ese entonces con aproximadamente 4 millones 552 mil personas, de las cuales 3 millones 211 mil constituían el padrón electoral (Comisión Estatal de Nuevo León, 2009; Gobierno del Estado de Nuevo León, 2010). En el proceso participaron cinco candidatos, aunque solo dos de ellos, Fernando Elizondo (Partido Acción Nacional [PAN]) y Rodrigo Medina (una coalición de partidos liderada por el Partido Revolucionario Institucional [PRI]), tenían posibilidades reales de ser electos. Los diarios y las televisoras locales, siguiendo el modelo de espectacularización e info-entretenimiento que ha caracterizado desde mediados de los años noventa a los medios informativos nacionales (Domínguez \& Lawson, 2004; Domínguez, Lawson \& Moreno, 2009; Esteinou, 2007; Lawson, 2008; Lozano, 2006), dedicaron gran parte de su cobertura electoral a los ataques, descalificaciones, las personalidades y la posición de los dos principales contendientes en los sondeos de opinión en lugar de centrarse en los temas y las propuestas. Así mismo, las estaciones de televisión llevaron a los candidatos y a sus esposas e hijos a programas de deportes y de entretenimiento o revista como invitados e incluso como conductores, dándole mayor preferencia a un candidato que a otro. Después de cinco meses de una cobertura electoral intensa y abrumadora (tres de campaña oficial pero dos más de atención previa), 1 millón 653 mil ciudadanos votaron el 5 de julio de 2009. Rodrigo Medina, de la coalición liderada por el PRI, resultó electo gobernador del Estado de Nuevo León para el período 2009-2015 con 49\% de los votos, contra $44 \%$ recibido por Fernando Elizondo. 
El presente trabajo no se enfoca en analizar las razones que tuvieron los votantes neoloneses para elegir un candidato sobre el otro, ni se interesa en analizar si la cobertura electoral favoreció a un candidato o fue equilibrada (algo que se exploró en un estudio paralelo). El propósito de este trabajo fue explorar la percepción y la evaluación de los votantes exclusivamente del área metropolitana de Monterrey sobre el desempeño de sus medios locales en la cobertura de la elección y su posición sobre la objetividad y la imparcialidad de las noticias acerca del proceso.

\section{MÉTODO}

Este estudio se basa en una encuesta telefónica probabilística a 400 votantes del área metropolitana de Monterrey, la cual comprende nueve ciudades: Monterrey, San Pedro Garza García, Santa Catarina, Guadalupe, San Nicolás de los Garza, Apodaca, General Escobedo y Juárez con una población total de 3 millones 700 mil personas. La encuesta fue realizada entre el 15 de junio y el 15 de julio de 2009, tres semanas antes y dos semanas después de la elección. Se seleccionaron únicamente a personas de 18 años o más que declararon tener credencial de elector. Los números telefónicos fueron obtenidos mediante un muestreo probabilístico por etapas del directorio telefónico 2007 de Teléfonos de México (Telmex) para el área metropolitana de Monterrey. En cada una de las 200 páginas del directorio se escogió aleatoriamente una de sus cuatro columnas. De cada una de ellas se seleccionaron, también de manera aleatoria, seis números telefónicos, los dos primeros como parte de la muestra y los otros cuatro como reemplazos para casos de rechazo o de números desconectados o sin servicio.

La mayoría de las preguntas de la encuesta se dedicaron a determinar la percepción y la evaluación de la cobertura electoral de la elección estatal por parte de los medios locales. El instrumento buscaba, a través de ellas, encontrar pistas sobre el tipo de decodificaciones generales de las noticias realizadas por los respondientes y sobre su nivel de escepticismo o distancia crítica acerca de la cobertura. Entre más baja fuera la calificación asignada a los medios y más baja su creencia en la objetividad e imparcialidad de los diarios y los noticieros televisivos, más activos y críticos se consideraron en sus lecturas. De acuerdo con Moores (2005), 
el significado de cualquier mensaje - en este caso una noticia electoral-se construye diferenciadamente dependiendo de la diversidad social entre los miembros de las audiencias. Agrega que individuos en diferentes posiciones dentro de la formación social, utilizando variados recursos interpretativos, dan sentido a los contenidos mediáticos en forma particular. Debido al sesgo clasista inherente a la disponibilidad de telefonía fija en México (solo existen 18 líneas por cada 100 habitantes), la clase social no fue incluida como variable en la encuesta. Sin embargo, el nivel educativo, aspecto que con frecuencia se relaciona con la clase social, sí fue incluido en el estudio, así como el género del votante y su edad. En el caso particular de la decodificación de noticias electorales, decidimos incluir la simpatía o ideología partidistas. En las sociedades contemporáneas, la afiliación o simpatía hacia un partido político -ya sea por razones familiares, tradicionales, corporativas o ideológicas- es clave para la interpretación última de las noticias electorales. Al incluir estas variables como indicadores de la pertenencia de los votantes a subgrupos diferentes, tuvimos en mente la advertencia de Morley (1985) de no considerar las categorías sociales como educación, género o edad como "correlatos o determinantes objetivos de las posiciones diferenciadas de decodificación" (p. 244). Para ello hubiésemos tenido que incluir otro tipo de datos - principalmente cualitativos- que permitieran especificar cómo esas variables intervienen realmente en el proceso de decodificación. Sin embargo, la premisa de este trabajo es que, a pesar de las obvias limitaciones de un estudio cuantitativo, analizar las relaciones entre estas categorías sociales y la percepción y evaluación de los medios informativos y su cobertura electoral permite generar un conocimiento modesto pero importante capaz de ir más allá de los estudios de caso para generalizarlo a una población más amplia.

\section{RESULTADOS}

¿Qué tan críticos fueron los votantes acerca de los medios informativos locales y su cobertura electoral?, ¿aceptaron o rechazaron las premisas o posiciones de las noticias acerca de los candidatos y el proceso mismo?, ¿fueron capaces de mantener una posición escéptica o negociada sobre los ángulos y enfoques particulares de cada diario o noticiero televisivo? 
De acuerdo al enfoque de los estudios culturales, las audiencias tienen "habilidades potenciales o efectivas y una pluralidad de formas para involucrarse con los mensajes y de incorporarlos en la vida real" (Liebes, 2005 , p. 365) permitiendo a las audiencias realizar lecturas negociadas de los contenidos mediáticos. Como explica Hall (1980), las estructuras de significación, conocimiento y relaciones sociales de los televidentes y lectores les permiten decodificar los significados de los medios e incluso rechazar parte de su carga ideológica desde una posición crítica.

La Tabla 1 muestra que los votantes regiomontanos se encontraban sorprendentemente satisfechos con sus medios informativos. Al preguntarles cómo evaluaban la cobertura del proceso electoral por cada uno de los diferentes medios locales con relación a su balance, imparcialidad y pluralismo, les asignaron calificaciones a sus medios de entre 8.2 y 9.0 en una escala de 0 a 10; una muy buena evaluación considerando la tradicional desconfianza de los mexicanos hacia la imparcialidad de los procesos electorales (De las Heras, 2006; Flores \& Meyenberg, 2000).

\section{Calificación por género}

A pesar de la importancia del género como una mediación social que frecuentemente explica diferencias en muchos procesos y situaciones, los votantes de sexo masculino y los de sexo femenino regiomontanos mostraban una satisfacción similar en la cobertura electoral en el caso de la mitad de los medios analizados (El Norte, Telediario matutino, Televisa Monterrey matutino y Televisa Monterrey nocturno), mostrando que en lo general esta variable no generaba percepciones diferenciadas entre las audiencias de estos medios. En los casos de Milenio Diario, Telediario nocturno e Info 7, sin embargo, las mujeres daban una mayor calificación que los hombres. En el caso del periódico, esto podía deberse a la baja cantidad de personas que reportaron leerlo con frecuencia (19 solamente) más que a percepciones distintas entre sus lectores de acuerdo al género. En el caso de Telediario nocturno, conducido por el arquitecto Héctor Benavides, un veterano de la televisión local, y de Info 7, cuyo titular era Luis Padua, la diferencia podría explicarse quizá por las personalidades de ambos (el primero recibió la máxima calificación en cuanto a credibilidad en otra de las preguntas del cuestionario; el segundo, más joven, recibió altas calificaciones por parte 


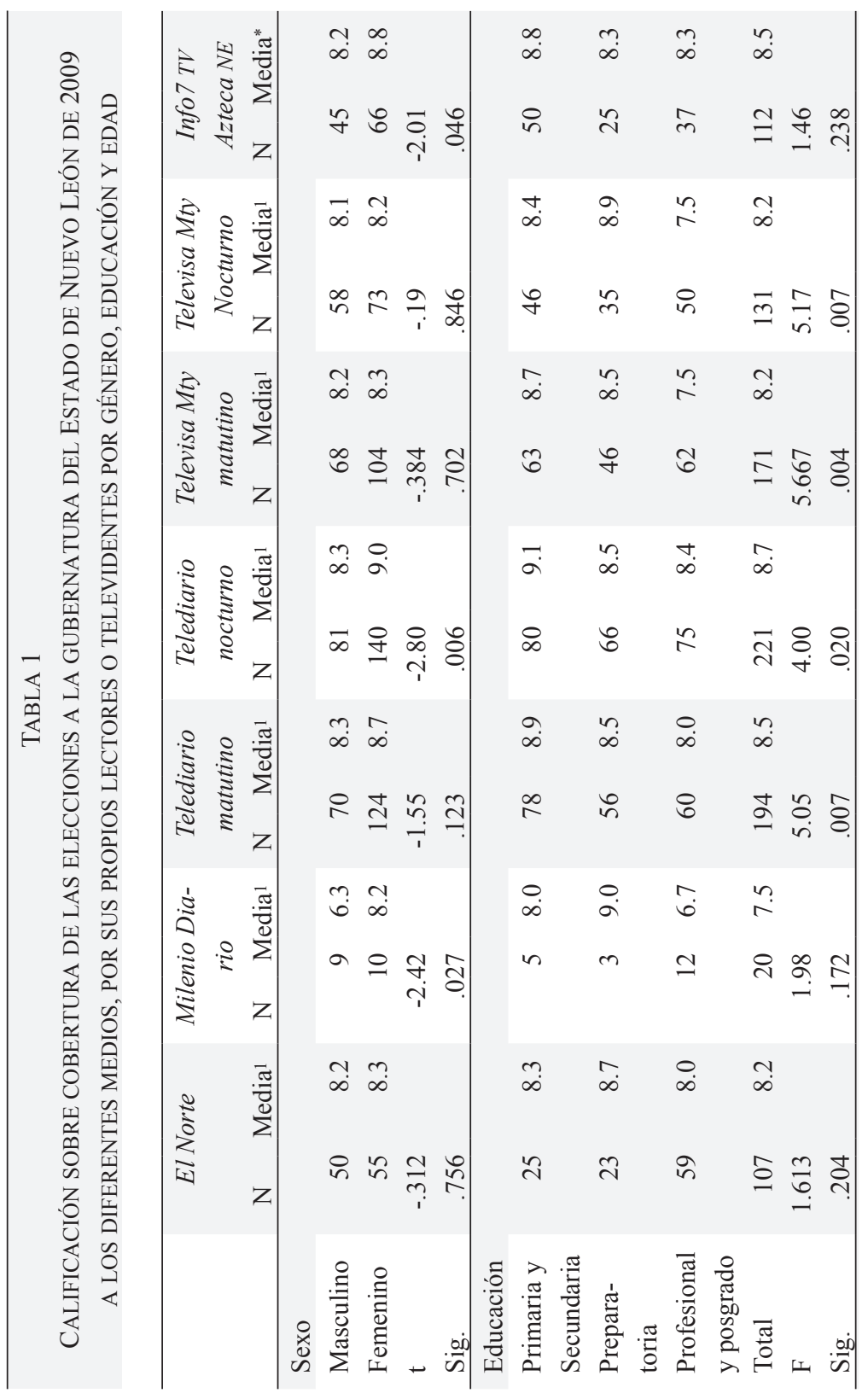




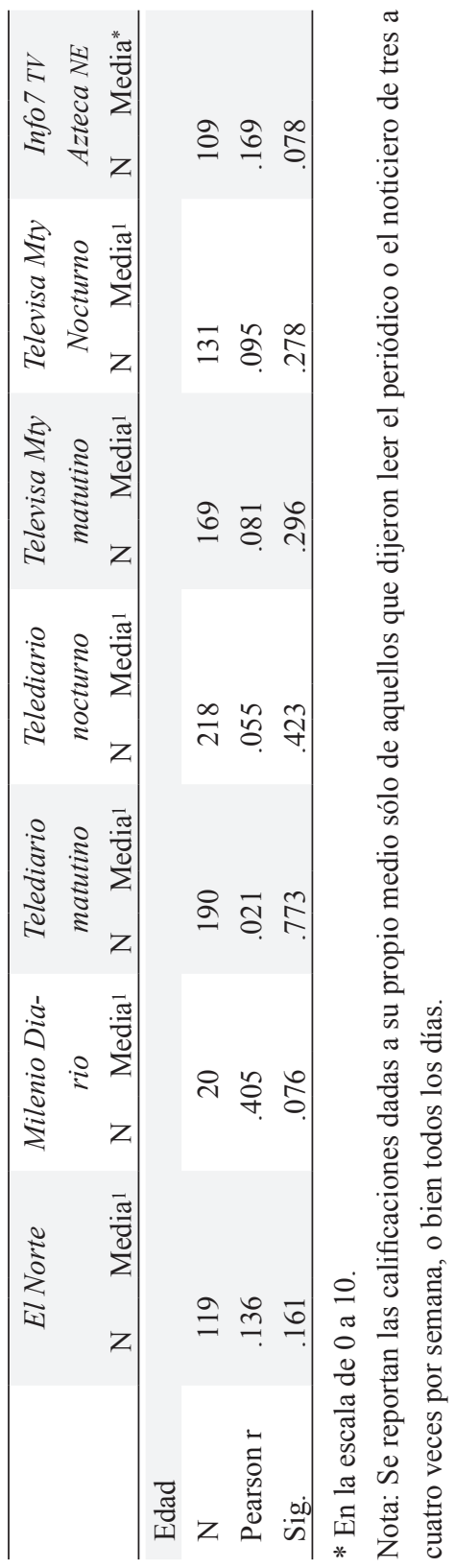


de los respondientes femeninos de la encuesta), pero no se recabaron explicaciones explícitas del por qué les otorgaban dicha calificación.

\section{Calificación por educación}

En contraste, el nivel educativo sí marcaba diferencias en la evaluación para el caso de los noticieros televisivos, aunque no para los periódicos. Los más educados daban calificaciones más bajas a los noticieros con mayores niveles de audiencia en el área metropolitana: las ediciones matutinas y nocturnas de Telediario de Multimedios y Las Noticias, de Televisa Monterrey, aunque no se presentaba el mismo caso en el noticiero Info 7, el programa que tenía menos cantidad de seguidores (véase Tabla 1). Así, la educación entendida como una mediación social fundamental, era un predictor útil del grado de satisfacción con la cobertura electoral de los noticieros televisivos más populares, aunque las calificaciones de entre 7.5 y 8.4 de quienes contaban con licenciatura o posgrado para sus medios electrónicos más vistos seguían siendo relativamente altas y diferenciándose en la práctica muy poco de los 8.4 a 9.1 de quienes solo contaban con primaria.

\section{Calificación por edad y simpatía partidista}

La edad no marcaba ninguna diferencia en los distintos medios. La simpatía partidista, por su parte, tampoco generaba diferencias en la percepción de qué tan bien había realizado su cobertura electoral el medio al que se exponían con frecuencia. Los panistas y los priístas evaluaban igual de bien a los medios informativos en los que se exponían sus contrapartes. La percepción de los votantes acerca del buen desempeño de sus medios informativos parecía ser bastante generalizada, con la excepción específica del nivel educativo para el caso de los noticieros televisivos.

\section{QUÉ TAN BALANCEADOS CONSIDERARON}

\section{A SUS MEDIOS INFORMATIVOS}

La mayoría de los votantes regiomontanos, así, parecía aceptar sin mayores cuestionamientos la proclamada adherencia de los medios a los que se exponía con frecuencia a los principios de balance, pluralidad e imparcialidad. 
Esta conclusión se matizaba al revisar las respuestas a otra pregunta relacionada: “¿Qué tan balanceados o partidistas considera usted que han sido los siguientes medios de comunicación, en lo que se refiere a la cobertura de los distintos partidos y candidatos a la gubernatura del Estado?". En este caso, la evaluación contrastaba drásticamente según se tratara del propio medio al que se exponía el votante o del resto de los medios a los que no estaba expuesto. La Tabla 2 muestra que los encuestados que se exponían de manera frecuente a un determinado medio tendían a considerar que había sido balanceado en su cobertura, a diferencia de quienes no eran lectores o televidentes asiduos del mismo. Mientras que $70 \%$ de sus propios lectores consideraban que el periódico El Norte había sido balanceado en su cobertura electoral, solamente $35 \%$ de los no lectores o lectores poco asiduos lo consideraban así. De la misma manera, $75 \%$ de sus propios televidentes consideraban balanceada la cobertura electoral de Telediario nocturno, contra solo $41 \%$ de los no televidentes. De los televidentes asiduos a Las Noticias matutino de Televisa Monterrey, 65\% pensaban que había sido balanceado en su información electoral, frente a $41 \%$ de quienes no lo veían o lo veían muy poco. Los hallazgos de la encuesta no permiten determinarlo de manera explícita, pero todo parece indicar que había una correlación en las audiencias regiomontanas entre confianza en un medio de comunicación y su exposición al mismo. Paralelamente, los resultados sugieren que quienes desconfiaban más de un medio, tendían a exponerse menos a él. Esta situación complica el conocer de manera objetiva el desempeño del medio de comunicación en la cobertura electoral de 2009, pues claramente la percepción de la audiencia dependía de su vinculación cotidiana con el periódico o el noticiero. ¿Quiénes estaban mejor posicionados para evaluar el desempeño de los medios regiomontanos?, ¿los propios lectores o televidentes del medio, por el seguimiento permanente de sus noticias, reportajes o entrevistas, o los no lectores o no televidentes del medio, más distanciados y por tanto más capaces de ver su desempeño de manera más clara? Lo que parece importar aquí, más allá de dar respuesta a esta pregunta, es identificar los patrones de comportamiento de los ciudadanos al percibir el desempeño de sus medios de comunicación y percatarse de cómo la exposición cotidiana a un medio de comunica- 


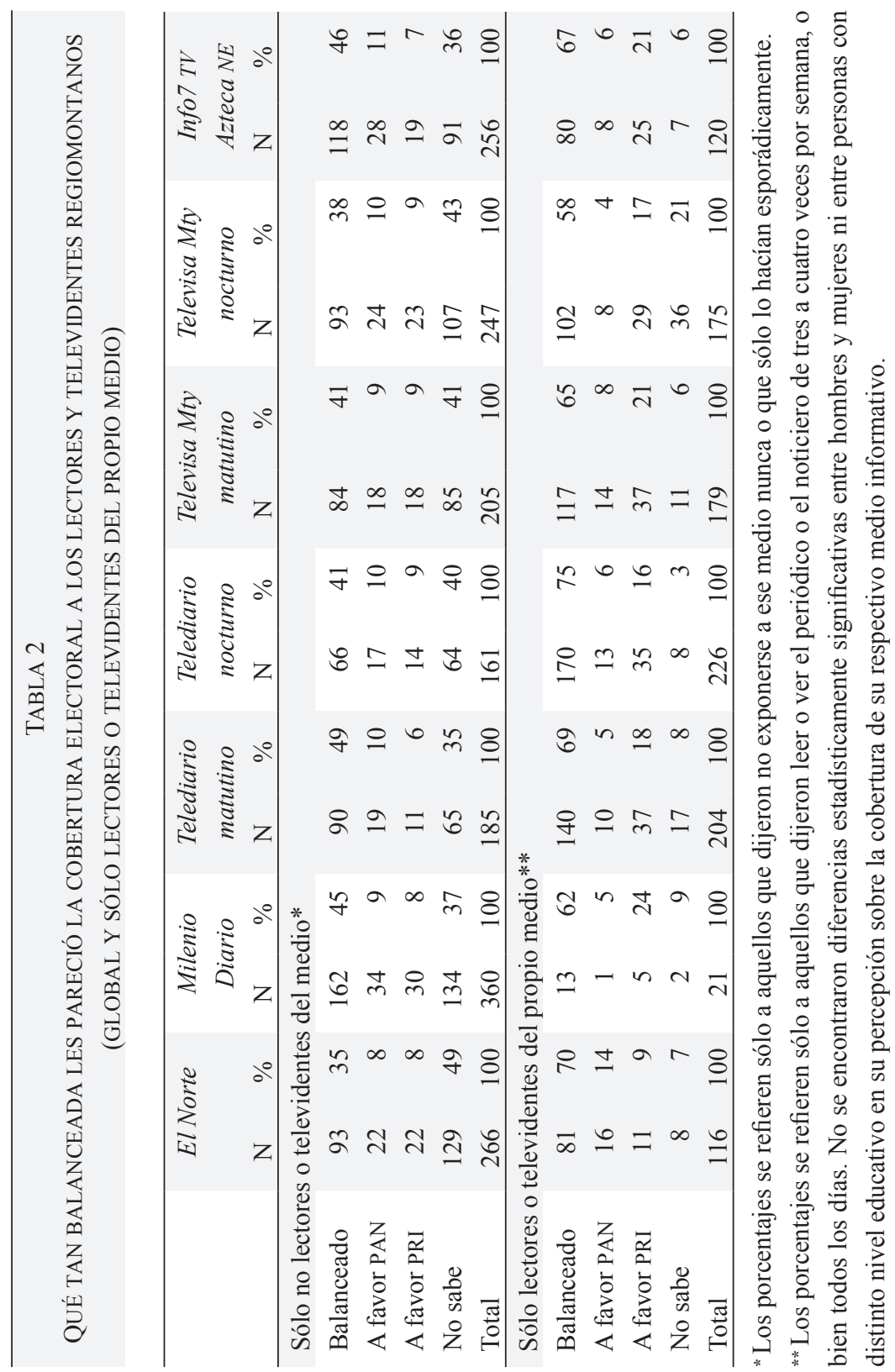


ción, o se debe a un convencimiento previo de su balance y objetividad, o genera a posteriori una lealtad que mueve al lector o televidente a percibir como más balanceado y objetivo a su medio.

Los medios mejor evaluados por su propia audiencia eran claramente los Telediario matutino y Telediario nocturno, así como el periódico El Norte, con porcentajes de 69 a 75\% en la categoría de "balanceados". Los medios peor evaluados por sus propias audiencias eran Televisa Monterrey nocturno, con 58\% percibiéndolo balanceado y Milenio Diario, con $62 \%$ en ese mismo rubro. Aún así, quedaba claro que más de la mitad de sus lectores o televidentes percibían favorablemente la cobertura electoral realizada en 2009. En contraste, ninguno de los medios analizados llegaba a la mitad de los encuestados en la opinión de que habían sido balanceados. Más de 50\% desconfiaba de su actuación o se sentía sin información suficiente para saberlo.

Sin embargo, como señala Lewis (1997), no nos debemos dejar llevar por las tendencias estadísticas cuando aplicamos métodos cuantitativos a fenómenos que guardan aspectos cualitativos importantes. Incluso entre los propios lectores o televidentes, de 25 a $42 \%$ consideraba que la cobertura electoral de su medio se había inclinado a favor de un partido u otro o no alcanzaba a discernir si había sido balanceada o no. Entre los votantes no lectores o televidentes del medio la situación era más dramática aún, pues esos porcentajes subían en algunos casos hasta $64 \%$.

Estos resultados dicen mucho sobre la cultura política de los regiomontanos. Mientras que según el Pew Research Center (2009), Estados Unidos ha registrado de 1985 a 2009 un descenso sistemático en la credibilidad de los medios informativos (60\% de los estadounidenses cree que están políticamente sesgados) a tono con lo sugerido por los partidarios del enfoque de estudios culturales que destacan la capacidad de las audiencias de evaluar críticamente y cuestionar a los medios, en Monterrey entre 7 y $21 \%$ aseguraba que habían favorecido al PAN o al PRI, abundando sin embargo los encuestados que declaraban no saber si habían sido equitativos o no (entre el 21 y $71 \%$ de los respondientes, dependiendo del medio).

Los hallazgos de la encuesta en Monterrey, tanto en la calificación que los votantes le dieron a los medios por su cobertura, como en su percepción acerca del grado de balance al informar, reflejan sin embar- 
go un fenómeno más universal, en el que los medios de comunicación, en general, son evaluados satisfactoriamente y por encima de las instituciones gubernamentales. De acuerdo a una encuesta en 10 países por parte de GlobeScan Incorporated (citado en Abundis, 2006), 61\% aprueba a sus medios de comunicación y en especial a la televisión, medio que se reporta de manera consistente como el más utilizado por la población para ser informados y el mejor calificado de todos en cuanto a credibilidad y confianza.

Una encuesta de Parametría realizada en marzo de 2006 en México (Abundis, 2006), unos meses antes de las polémicas elecciones presidenciales y en plena campaña electoral, encontró que $48 \%$ de la población afirmaba que los medios eran confiables y $29 \%$ que eran más o menos confiables. Solo $19 \%$ pensaba que no lo eran. El medio más confiable era la televisión, con 7 de cada 10 personas declarando que les generaba mucho o algo de confianza. No era claro si al nivel cualitativo había matices en estas opiniones cuantitativas. Sin embargo, estos hallazgos apuntan hacia la importancia y la relevancia que los medios, y en particular la televisión, tienen en la vida cotidiana de los ciudadanos en todo el mundo y qué tan cercanos y creíbles son para ellos. Al parecer, la cercanía con los medios y la integración de los mismos dentro de sus rutinas y prácticas sociales, dificultan a los votantes distanciarse críticamente y cuestionar sus posiciones ideológicas y coberturas electorales.

\section{BAJO INTERÉS EN LA POLÍTICA}

Podría concluirse que una de las razones de esta aparente ausencia de percepciones críticas hacia los medios informativos en los procesos electorales sería el bajo nivel de interés de los votantes regiomontanos en la política. De acuerdo a la encuesta, 63\% de los respondientes declararon estar "poco interesados en la política" y 17\% adicional señaló que no le interesaba nada: un total de $80 \%$ de los encuestados, sin diferencias significativas por género, educación o simpatía partidista (véase tablas 3 y 4). Estas mediaciones sociales, a pesar de su relevancia al separar a la muestra en grupos y subgrupos, no marcaban diferencias en el grado de interés o desinterés de los respondientes en la política, sugiriendo la posibilidad de que dicha ausencia de interés sobre la política y 
los políticos había trascendido cualquier dimensión sociodemográfica. Estos hallazgos son más altos que el promedio general para la población mexicana detectado por la Encuesta de Cultura Política de 2008, que encontró que solo 9.3\% de los mexicanos en general manifestaba estar muy interesado en la política (Secretaría de Gobernación, 2008), pero 8 de cada 10 regiomontanos seguían mostrando escaso interés en el tema. Quizá, como argumenta Buckingham (1999) para el caso de la falta de interés en la política de los adolescentes británicos, los votantes regiomontanos muestran desinterés en la política porque están y se sienten excluidos del dominio de la misma y las formas dominantes del discurso político, por sentirse sin representación de sus intereses. Otra posibilidad, como menciona Brunner (1997) es que las encuestas sobre interés en la política no capten adecuadamente la dimensión más profunda y compleja de la conciencia que aplica, "donde las personas sí poseen considerables conocimientos sobre dicha realidad y son competentes para formarse juicios sobre ella" (p. 4). Como explican Dahlgren y Gurevitch (2005), esos datos representarían no un desinterés en la política en sí misma, sino un reenfocamiento de la participación política, o la emergencia de nuevas definiciones de lo que constituye lo político caracterizadas por "una participación personalizada más que colectiva, y un mayor énfasis en temas específicos e individuales que en plataformas o ideologías generales" (pp. 377-378).

¿Son más propensos los ciudadanos interesados en la política a realizar evaluaciones más críticas de la cobertura electoral? El análisis de varianza en una dirección sobre el grado de interés en la política y la calificación tanto de la cobertura como del balance de los medios informativos locales en una escala de 0 a 10 no alcanzó diferencias significativas entre quienes estaban muy interesados y poco interesados en la política. Pareciera que en el caso de los votantes regiomontanos existía un consenso, sin importar su grado de interés en la política, con respecto de la credibilidad y el balance de sus medios informativos preferidos. Ese $20 \%$ de encuestados que declaraba estar muy interesado en la política evaluaba igual de satisfactoriamente a sus medios que los poco o nada interesados. Esto contrasta con estudios previos en otras partes del mundo que han encontrado una asociación entre el grado de interés en la política y la visión crítica de la cobertura (véase por ejemplo 
TABLA 3

PORCENTAJE DEL GRADO DE INTERÉS EN LA POLÍTICA (GLOBAL)

\begin{tabular}{lrr}
\hline & \multicolumn{2}{c}{ Total } \\
\hline & $\mathrm{N}$ & $\%$ \\
\hline Mucho & 81 & 21 \\
Poco & 247 & 63 \\
Nada & 65 & 16 \\
Total & 393 & 100 \\
\hline
\end{tabular}

TABLA 4

PORCENTAJE DEL GRADO DE INTERÉS EN LA POLÍTICA POR GÉNERO Y EDUCACIÓN

\begin{tabular}{|c|c|c|c|c|c|c|}
\hline & \multicolumn{2}{|c|}{$\begin{array}{c}\text { Primaria a } \\
\text { Preparatoria }\end{array}$} & \multicolumn{2}{|c|}{$\begin{array}{c}\text { Licenciatura o } \\
\text { posgrado }\end{array}$} & \multicolumn{2}{|c|}{ Total } \\
\hline & $\mathrm{N}$ & $\%$ & $\mathrm{~N}$ & $\%$ & $\mathrm{~N}$ & $\%$ \\
\hline \multicolumn{7}{|l|}{ Masculino } \\
\hline Mucho & 10 & 13 & 21 & 29 & 31 & 21 \\
\hline Poco o nada & 68 & 87 & 52 & 71 & 120 & 79 \\
\hline Total & 78 & 100 & 73 & 100 & 151 & 100 \\
\hline $\mathrm{X}^{2}$ & 5.877 & & & & & \\
\hline Sig. & .015 & & & & & \\
\hline \multicolumn{7}{|l|}{ Femenino } \\
\hline Mucho & 31 & 20 & 18 & 23 & 49 & 21 \\
\hline Poco & 127 & 80 & 60 & 77 & 187 & 79 \\
\hline Total & 158 & 100 & 78 & 100 & 236 & 100 \\
\hline $\mathrm{X}^{2}$ & .379 & & & & & \\
\hline Sig. & .538 & & & & & \\
\hline
\end{tabular}

Iyengar, en Bregman, 1998). Quizás existe una relación más intensa y compleja de las audiencias regiomontanas con sus medios informativos locales que la existente en el resto del país. De hecho, a diferencia de muchas otras plazas donde los programas de mayor penetración son las telenovelas y otros contenidos nacionales, en Monterrey históricamente el primer lugar de audiencia lo han obtenido consistentemente los no- 
TABLA 5

PORCENTAJE DEL GRADO DE INTERÉS EN LA POLÍTICA POR SIMPATÍA PARTIDISTA

\begin{tabular}{lcc|cc|cc|cc}
\hline & $\begin{array}{c}\text { Simpatizante } \\
\text { PAN }\end{array}$ & \multicolumn{2}{c|}{$\begin{array}{c}\text { Simpatizante } \\
\text { PRI }\end{array}$} & Independiente & \multicolumn{2}{c}{ Total } \\
\hline & $\mathrm{N}$ & $\%$ & $\mathrm{~N}$ & $\%$ & $\mathrm{~N}$ & $\%$ & $\mathrm{~N}$ & $\%$ \\
\hline Mucho & 32 & 25 & 16 & 15 & 23 & 21 & 71 & 21 \\
Poco o & 98 & 75 & 88 & 85 & 85 & 79 & 271 & 79 \\
Nada & & & & & & & & \\
Total & 130 & 100 & 104 & 100 & 108 & 100 & 342 & 100 \\
$\mathrm{X}^{2}$ & 3.020 & & & & & & & \\
Sig. & .221 & & & & & & & \\
\hline
\end{tabular}

Nota: Entre los simpatizantes de cada partido o los independientes tampoco había diferencias estadísticamente significativas al controlar su interés en la política por género o educación.

ticieros locales. Así mismo, hay que recordar el éxito nacional del periodismo regiomontano. Además de su fortaleza e influencia en el área metropolitana de Monterrey, El Norte vino a modificar el panorama y el estilo de hacer periodismo en México al abrir diarios en Ciudad de México (Reforma) y en Guadalajara (Mural). De igual forma, el Grupo Multimedios, también originario de Monterrey, se ha vuelto un jugador importante en el periodismo impreso con sus 12 ediciones de Milenio en distintas regiones del país, sus numerosas estaciones radiofónicas y televisivas en 17 ciudades del norte de México. Es en estos resultados empíricos donde las limitaciones de un enfoque cuantitativo impiden determinar las razones y motivaciones más sutiles y complejas que podrían estar en juego, destacando la necesidad de un enfoque cualitativo complementario.

\section{CONCLUSIONES}

Los estudios sobre la credibilidad de las noticias electorales y su importancia para los votantes han sido escasos en América Latina y en particular en México. Mediante una encuesta telefónica a votantes en 
las elecciones estatales de Monterrey en 2009, este trabajo ofrece información sobre el grado de satisfacción que los votantes presentaron sobre la cobertura electoral de sus medios informativos locales.

En contraste con investigaciones que han encontrado evidencias de actividad y capacidad crítica en las audiencias de medios, este trabajo no encontró en lo general signos de insatisfacción con la cobertura ni evidencias de que los votantes advirtieran sesgos partidistas en sus periódicos o noticieros televisivos locales. Dadas las limitaciones de los datos cuantitativos para entrar a dimensiones más profundas, estos hallazgos no prueban que los lectores y televidentes fueran pasivos ni que fueran incapaces de negociar la información electoral, pero sugieren que en lo general los votantes estaban bastante satisfechos con la cobertura de sus medios.

Los hallazgos de este estudio son contrastantes. Por un lado, los respondientes parecían ser un tanto pasivos y acríticos si nos centramos en su confianza optimista sobre la cobertura electoral a los medios informativos locales, otorgándoles calificaciones muy altas en cuanto a objetividad e imparcialidad pese a los sesgos y tendencias partidistas en muchos de ellos (confirmadas en el análisis de contenido realizado por separado). ${ }^{3}$ Por otro lado, esa evaluación positiva no se correspondía con un interés profundo en el proceso electoral. Dado su poco interés en el proceso y su apatía hacia él, parecía extraño que fueran tan vocales y estuviesen tan convencidos sobre el buen desempeño de sus medios informativos locales al cubrir el proceso electoral. Como señala Dahlgren (2006), no debemos comparar a ciudadanos reales contra "el ideal del ciudadano responsable y comprometido que se impuso en los inicios del siglo XX" (p. 270). Necesitamos repensar la propia noción de ciudadanía y reconsiderar sus parámetros. En este caso particular, debemos empezar por preguntar sobre las razones subyacentes por las que los votantes regiomontanos parecen tener tanta confianza en sus medios locales y los significados más subjetivos y emocionales que les asignan a ellos cuando buscan información u orientación. Más aún, necesitamos entender que, contrario a las dinámicas habermasianas ideales de la democracia deliberativa, que asumen que los ciudadanos tienen la misma posibilidad de

3 Esto también ha sido detectado en el nivel regional y nacional por, Hughes y Lawson (2004). 
participar y de discutir en la esfera pública que los actores políticos y periodísticos, los procesos electorales reales son desordenados, asimétricos y desiguales, proporcionando a los votantes en este caso particular menos recursos reales y simbólicos para enfrentar a los medios y a las estrategias electorales de los candidatos. Como ha explicado Hall (1980), los contenidos mediáticos serán polisémicos, pero no tienden a ser balanceados y plurales; la encodificación establece los parámetros y los límites dentro de los cuales opera la decodificación (p. 172).

Estudios futuros dentro de esta línea de investigación deberán buscar más indicadores cuantitativos sobre la aceptación, negociación o rechazo de los encuadres noticiosos electorales y la participación de factores sociales en esas lecturas, algo que representa un desafío dadas las limitaciones metodológicas del enfoque cuantitativo para este tipo de aproximaciones. Así mismo, sería beneficioso adoptar una metodología más ambiciosa, combinando técnicas cuantitativas y cualitativas de investigación para poder interpretar y contrastar los hallazgos generalizables de las primeras con la comprensión más de fondo que ofrecen las segundas. Como ha notado Lewis (1997), aunque los estudios culturales tienen mucho que ganar si usan herramientas cuantitativas, no debemos de olvidar que éstas son más útiles para documentar la conducta que los significados subjetivos. Una aportación importante sería, siguiendo la sugerencia de Buckingham (1999), explorar empíricamente cómo la información política permite a los miembros de las audiencias construir y definir su relación con la esfera pública, en lugar de enfocarse solo en su habilidad para ser críticos o para negociar contenidos políticos específicos. En un contexto político como el que prevalece en México, donde la democracia no acaba por consolidarse $\mathrm{y}$ donde los medios informativos tienden a ser partidistas y sesgados, es imperioso explorar más detalladamente los patrones de recepción y apropiación de las noticias electorales por parte de los votantes.

\section{Bibliografía}

Abbe, O. G., Goodliffe, J., Herrnson, P. S. \& Patterson, K. D. (2003).

Agenda setting in congressional elections: The impact of issues and campaigns on voting behavior. Political Research Quarterly, 56 (4), 419-430. 
Abundis, F. (2006). Los medios de comunicación en México. Revista AMAI. Recuperado el 23 de enero de $2011 \mathrm{de} \mathrm{http://www.amai.org/}$ pdfs/revista-amai/AMAI-13_art8.pdf

Bennett, S. E., Rhine, S. L., \& Flicksinger, R. S. (2004). The things they cared about: Change and continuity in American attention to different news stories 1989-2002. The International Journal of Press/ Politics, 9 (1), 75-99.

Bregman, D. (1998). La función de agenda: una problemática en transformación. En J. Ferry, D. Wolton y et al. (Comps.), El nuevo espacio público. Madrid, España: Gedisa.

Brunner, J. J. (1997, 22 de julio). Ciudadanía y participación: notas para la discusión. Recuperado el 17 de septiembre de 2011 de http:// mt.educarchile.cl/mt/jjbrunner/archives/Ciud+Part.pdf

Buckingham, D. (1999). Young people, politics and news media: Beyond political socialisation. Oxford Review of Education, 25 (1-2), 171-184.

Cantú, J., Lozano, J. C., Martínez, F. J. \& Smith, C. (2011). Elecciones y medios de comunicación locales: el caso de la prensa y la televisión en las elecciones a la gubernatura de Nuevo León en 2009. Manuscrito.

Colwell, R. (1979). Mass media use and voting behavior: The accuracy of political perception among first-time and experienced voters. Communication Research, 6 (4), 407-436.

Comisión Estatal de Nuevo León (2009). Lista nominal por municipios. Recuperado el 28 de noviembre de 2009 de http://www.cee-nl.org. $\mathrm{mx} /$ listanominal.asp\#.

Dahlgren, P. (2006). Doing citizenship: The cultural origins of civic agency in the public sphere. European Journal of Cultural Studies, 9 (3), 267-286.

Dahlgren, P. \& Gurevitch, M. (2005). Political communication in a changing world. En J. Curran \& M. Gurevitch (Eds.), Mass media and society (pp. 375-393). Londres, Ingalterra: Hodder Arnold.

David, C. C. (2009). Learning political information from the news: A closer look at the role of motivation. Journal of Communication, 59 (2), 243-261. doi: 10.1111/j.1460-2466.2009.01414.x

De las Heras, M. (2006). Por quién vamos a votar y por qué. México: Nuevo Siglo Aguilar. 
De Vreese, C. H. (2004). The effects of frames in political television news on issue interpretation and frame salience. Journalism \& Mass Communication Quarterly, 81 (1), 36-52.

Domínguez, J. I. \& Lawson, C. H. (2004). Mexico's pivotal democratic election: Candidates, voters, and the presidential campaign of 2000. Stanford, CA, EE.UU.: Stanford University Press.

Domínguez, J. I., Lawson, C. H. \& Moreno, A. (2009). Consolidating Mexico's democracy: The 2006 presidential campaign in comparative perspective. Baltimore, MD, EE.UU.: Johns Hopkins University Press.

Esteinou, J. (2007). La videocracia mexicana y las elecciones presidenciales de 2006. En L. Escudero (Ed.), Democracias de opinión: medios y comunicación política en las elecciones mexicanas 2006 (pp. 49-70). Buenos Aires, Argentina: La Crujía.

Eveland, W. P., Shah, D. V., \& Kwak, N. (2003). Assessing causality in the cognitive mediation model: A panel study of motivations, information processing, and learning during campaign 2000. Communication Research, 30 (4), 359-386. doi: 10.1177/0093650203253369

Farnsworth, S. J., \& Lichter, S. R. (2007). The nightly news nightmare: Television's coverage of U.S. presidential elections, 1988-2004 (2 ed.). Lanham, MD, EE.UU.: Rowman \& Littlefield Publishers.

Flores, J. \& Meyenberg, Y. (2000). Ciudadanos y cultura de la democracia: reglas, instituciones y valores. Encuesta nacional. México: UNAM/IFE.

Gauntlett, D. \& Hill, A. (1999). TV living: Television, culture, and everyday life. Londres, Inglaterra: Routledge.

Gobierno del Estado de Nuevo León (2010). Estadísticas. Recuperado el 5 de mayo de 2010 de http://www.nl.gob.mx/?P=nl_poblacion

Graber, D. A. (1993). Processing the news: How people tame the information tide. Lanham, MD, EE.UU.: University Press of America.

Hacker, K., Coste, T. G., Kamm, D. \& Bybee, C. (1991). Opositional readings of network TV news. Discourse and Society, 2 (2), 183-202.

Hall, S. (1980). Encoding/Decoding. En D. H. Stuart Hall, Andrew Lowe \& Paul Willis (Ed.), Culture, Media, Language. Londres, Inglaterra: Hutchinson.

Hermes, J. (2005). Re-reading popular culture. Malden; Oxford: Blackwell Pub. 
Hernández, M. A. (2008). La democracia mexicana, presa de una cultura política con rasgos autoritarios. Revista Mexicana de Sociología, 70 (2), 261-303.

Hollander, B. A. (2007). Media use and political involvement. En R. W. Preiss, B. M. Gayle, N. Burrell, M. Allen \& J. Bryant (Eds.), Mass media effects research: Advances through meta-analysis (pp. 377-390). Nueva York, EE.UU.: Lawrence Erlbaum Associates, Publishers.

Hughes, S. \& Lawson, C. (2004). Propaganda and crony capitalism: Partisan bias in Mexican television news. Latin American Research Review, 39 (3), 81-105.

Jensen, K. B. (1988). News as social resource: A qualitative empirical study of the reception of Danish television news. European Journal of Communication, 3, 275-301.

Jensen, K. B. (1998). News of the world: World cultures look at television news. Londres; Nueva York: Routledge.

Lawson, C. (2008). Election coverage in Mexico: Regulation meets crony capitalism. En J. Strömbäck \& L. L. Kaid (Eds.), The handbook of election news coverage around the world. Nueva York, EE.UU.: Routledge.

Lewis, J. (1997). What counts in cultural studies. Media Culture \& Society, 19 (1), 83-97.

Liebes, T. (2005). Viewing and reviewing the audience: Fashions in communication research. En J. Curran \& M. Gurevitch (Eds.), Mass media and society (pp. 356-374). Londres, Inglaterra: Hodder Arnold.

Livingstone, S. M. (Ed.). (2005). Audience and publics: When cultural engagement matters for the public sphere. Portland, OR, EE.UU.: Intellect Books.

Lozano, J. C. (2006). Political advertising in Mexico. En L. L. Kaid \& C. Holtz-Bacha (Eds.), The Sage handbook of political advertising (pp. 259-268). Thousand Oaks, CA, EE.UU.: Sage.

Madianou, M. (2005). The elusive public of television news. En S. Livingstone (Ed.), Audiences and publics: When cultural engagement matters for the publich sphere (pp. 99-114). Bristol, Inglaterra: Intellect.

Moores, S. (2005). Media/theory: thinking about media and communications. Londres Nueva York: Routledge. 
Morley, D. (1980). The "Nationwide” audience: Structure and decoding. Londres, Inglaterra: British Film Institute.

Morley, D. (1985). Cultural transformations: The politics of resistance. En M. Gurevitch \& M. R. Levy (Eds.), Mass Communication Review Yearbook (pp. 237-250). Newbury Park, CA, EE.UU.: Sage.

Nadeau, R., Nevitte, N., Gidengil, E., \& Blais, A. (2008). Election campaigns as information campaigns: Who learns what and does it matter? Political Communication, 25 (3), 229-248. doi: 10.1080/10584600802197269

Orozco, G. (1997). La televidencia de lo político: un complejo proceso de mediaciones. Oficios Terrestres (4), 18-21.

Orozco, G. \& Medina Jackson, D. (2000). Supertemas noticiosos en la televisión mexicana. Análisis de la información en los noticiarios 24 Horas y Hechos. Comunicación y Sociedad (37), 53-86.

Pew Research Center (2009). Public evaluations of the news media: 1985-2009. Recuperado el 15 de marzo de 2010 de http://www. people-press.org

Putnam, R. D. (2000). Bowling alone: The collapse and revival of American community. Nueva York, EE.UU.: Simon \& Schuster.

Rhee, J. W., \& Cappella, J. N. (1997). The role of political sophistication in learning from news: Measuring schema development. Communication Research, 24 (3), 197-233.

Sartori, G. (1998). Homo videns: la sociedad teledirigida. Madrid, España: Taurus Ediciones.

Scammell, M. \& Semetko, H. A. (2008). Election news coverage in the U.K. En J. Strömbäck \& L. L. Kaid (Eds.), The handbook of election news coverage around the world. Nueva York, EE.UU.: Routledge.

Secretaría de Gobernación (2008). Encuesta Nacional sobre Cultura Política y Prácticas Ciudadanas. Recuperado el 28 de noviembre de 2009 de: http://www.encup.gob.mx/en/Encup/Cuarta_ENCUP_2008

Sigman, S. J. \& Fry, D. L. (1985). Differential ideology and language use: Readers' reconstructions and descriptions of news events. Critical Studies in Media Communication, 2 (4), 307-322.

Strömbäck, J. \& Kaid, L. L. (2008). A framework for comparing election news coverage around the world. En J. Strömbäck \& L. L. Kaid 
(Eds.), The handbook of election news coverage around the world. Nueva York, EE.UU.: Routledge.

Tenenboim-Weinblatt, K. (2009). "Where is Jack Bauer when you need him?". The uses of television drama in mediated political discourse. Political Communication, 26 (4), 367-387. doi: 10.1080/10584600903296960

Van Zoonen, L. (2007). Audience reactions to Hollywood politics. Media, Culture \& Society, 29 (4), 531-547.

Van Zoonen, L. (2005). Entertaining the citizen: When politics and popular culture converge. Lanham, MD, EE.UU.: Rowman \& Littlefield.

Van Zoonen, L. (2004). Imagining the fan democracy. European Journal of Communication, 19 (1), 39-52.

Weaver, D. \& Drew, D. (2001). Voter learning and interest in the 2000 presidential election did the media matter? Journalism \& Mass Communication Quarterly, 78 (4), 787-798.

Fecha de recepción: 29/04/2011. Aceptación: 08/09/2011. 